\title{
ESTIMATIVA DO DAP EM FUNÇÃO DO DIÂMETRO DO TOCO PARA PLANTIOS DE Eucalyptus urograndis IMPLANTADOS EM DIFERENTES ESPAÇAMENTOS
}

Plínio Carielo, Renato de Araújo Ferreira, João Luiz DalPonte Filho, Gabrielle Bonifácio da Silva, Luiz Paulo Leandro da Silva, Thamirys Serafim.

Centro Estadual de Educação Tecnológica Paula Souza, Escola Técnica Estadual Prof. Dr. Antonio Eufrásio de Toledo - ETEC, Curso Técnico em Florestas, Presidente Prudente, SP. E-mail: pliniocarielo@hotmail.com

\section{RESUMO}

Um dos principais objetivos dos levantamentos florestais comerciais é a determinação do volume de madeira. Entretanto há situações que as condições originais da floresta foram alteradas, inclusive cortadas; necessitando da reconstituição das condições iniciais do povoamento florestal, para determinar a produção total que não foram registradas. Para reconstituir estes dados utilizase a análise de regressão, técnica empregada pela dendrometria, que relaciona o volume da madeira, que de fato é uma variável de difícil obtenção. Por outro lado, há variáveis mais facilmente mensuráveis e consequentemente mais baratas como é o caso do diâmetro, da altura do peito (DAP) e da altura total (HT). Diante disso este trabalho ajustou diferentes modelos matemáticos para estimar o DAP em função do diâmetro do toco e encontrou boas estatísticas de ajuste e precisão para ambos os modelos testados demonstrando aplicabilidade desta técnica.

Palavras - Chave: Manejo Florestal, Regressão, Relações Dendrometricas, Estatísticas de Ajuste e Precisão.

\section{ESTIMATED DAP ACCORDING TO THE DIAMETER OF THE FOR PLANTATIONS OF Eucalyptus urograndis IMPLEMENTED IN DIFFERENT SPACING}

\begin{abstract}
One of the main objectives of commercial forest surveys is to determine the volume of wood. However there are situations that the original forest conditions have been changed, even cut requiring reconstitution of the initial conditions of afforestation, to determine the total production that were not recorded. To recover these data it is used a regression analysis, a technique used by dendrometry, that relates the volume of wood, in fact it is a variable very difficult to obtain. On the other hand, there are easily measurable variables and consequently cheaper such as the diameter, the breast height (DBH) and total height $(\mathrm{HT})$. In addition this work set different mathematical models to estimate the DAP according to the stump diameter and found good fit accuracy statistics for both models tested demonstrating applicability of this technique.
\end{abstract}

Keywords: Forest Management, Regression, dendrometric Relations, Adjustment Statistics and Precision Statistics. 


\section{INTRODUÇÃO}

Um dos principais objetivos dos levantamentos florestais comerciais é a determinação do volume de madeira, em metros cúbicos, existente numa determinada área de floresta por meio de suas variáveis dendrométricas que podem ser facilmente medidas ou estimadas por instrumentos florestais ou por relações dendrométricas.

As variáveis dendrométricas mais utilizadas nestes levantamentos florestais são o Diâmetro a altura do peito (DAP) e altura total (HT) das árvores. Estas variáveis são classificadas como sendo medidas direta e indireta, respectivamente (SOARES, et al., 2006). O DAP é uma medida direta pois ao coletarmos esta variável com uma suta florestal entramos em contato com a árvore; já a HT realizamos uma estimativa por meio de instrumentos, hipsômetros, que não entram em contato com a árvore.

Quando trabalhamos com variáveis que são coletadas indiretamente, é pratica usual não medir todas as árvores e utilizar uma amostra para gerar equações matemáticas que permitem a determinação de uma variável com base em outra variável de medição direta, ou seja, criação de uma relação dendrométrica. Estas equações matemáticas, relações, são muito utilizadas na área florestal seja para determinar a HT por meio do DAP, denominadas de equações hipsométricas; ou volume sólido $\left(\mathrm{m}^{3}\right)$ por meio do DAP e HT, denominadas de equações volumétricas.

O desenvolvimento das relações dendrométricas tem sido feito para facilitar os trabalhos do inventário florestal (técnica existente para quantificar o volume de florestas), intimamente vinculado à teoria da amostragem (PÉLLICO NETTO \& BRENA, 1997 citados por NASCIMENTO, et al., 2010). Assim os profissionais da área florestal têm criado essas relações previamente à realização do inventário florestal para tornar esta atividade mais rápida e menos onerosa (NASCIMENTO, et al., 2010).

Existem ainda outras situações práticas no dia a dia dos profissionais ligados à área florestal onde as condições originais da floresta foram parcialmente ou totalmente alteradas, ou seja, cortadas; necessitando da reconstrução das condições iniciais do povoamento florestal, para determinar a produção total que não foram registradas, ou para determinar o volume de madeira removido ilegalmente por invasores, para fins de cobrança judicial ou para reconstituir uma área desmatada e permitir a compensação ambiental (URBAN, et al., 2010).

Para reconstituir estes dados a Ciência Florestal utiliza-se de técnicas e conhecimentos gerados pela "Dendrometria" ciência que segundo Machado e Filho (2006) estuda, pesquisa e desenvolve métodos e técnicas para determinar as dimensões, volume, idade, crescimento e peso de árvores em pé ou derrubadas.

Umas das técnicas empregadas pela Dendrometria é a regressão linear que relacionam uma variável de difícil obtenção, como o volume, por exemplo, com variáveis mais facilmente mensuráveis e consequentemente mais baratas como o DAP e a HT. Tais relações são obtidas por meio da análise de regressão, onde ajusta se um modelo matemático com dados de árvores remanescentes representativas da população.

Segundo Leite (2008) é possível estimar o volume de árvores em áreas de floresta de terra firme que foram desmatadas ilegalmente e que não existe informações sobre o volume de madeira colhido, medindo-se apenas o diâmetro das cepas (tocos) que ficaram na área após a exploração. Leite (2008) diz ainda que existe uma boa relação entre volume e diâmetro da cepa e que a precisão de equações volumétricas geradas a partir do diâmetro da cepa se compara a precisão de equações volumétricas geradas somente em função do DAP, considerando um nível de significância de $5 \%$ pelo teste t.

Diante disso este trabalho buscou ajustar diferentes modelos matemáticos, para estimar o DAP, que permite o cálculo de outras variáveis, como Altura e Volume; em função do diâmetro do toco, em virtude do toco ser a única parte do tronco que permanece no campo após o corte e retirada da árvore; utilizando se para isso dados de povoamentos clonais de Eucalyptus urophylla 
vs. Eucalyptus grandis implantados em diferentes espaçamentos na região de Presidente Prudente, SP.

\section{METODOLOGIA}

Localização e caracterização da área.

O presente trabalho foi realizado na ETEC Prof. Dr. Antonio Eufrásio de Toledo, localizada na Rodovia Raposo Tavares Km 561, bairro Nova Prudente, cujas coordenadas geográficas são de 2210'54" S e 5122'01" O.

As árvores mensuradas pertencem a reflorestamentos comerciais implantados em 2014 com diferentes espaçamentos, 3, 6 e $12 \mathrm{~m}^{2} /$ planta, nos arranjos $3 \times 1 \mathrm{~m}, 3 \times 2 \mathrm{~m}$ e $3 \times 4 \mathrm{~m}$, respectivamente.

\section{Dados coletados}

Foram coletados o Diâmetro a Altura do Peito (DAP - 1,30m do solo) e o Diâmetro do Toco (Dtoco - $5 \mathrm{~cm}$ do solo) de 50 indivíduos de cada reflorestamento com auxílio de uma suta florestal.

\section{Avaliações realizadas}

Os dados dos diâmetros a altura do peito e diâmetro do toco de 40 árvores mensuradas foram utilizados para ajustar quatro modelos matemáticos, a fim de determinar equações matemáticas para estimar os diâmetros a altura do peito das outras 10 árvores mensuradas para validação das equações matemáticas ajustadas por meio do Teste de Comparação de Médias de Tukey a 5\% de probabilidade por meio do programa estatístico denominado Assistat.

Os modelos ajustados foram:
a) $\mathrm{DAP}=\mathrm{b} 0+\mathrm{b} 1 *$ Dtoco
c) $\mathrm{DAP}=\mathrm{b} 0+\mathrm{b} 1 *$ Dtoco $+\mathrm{b} 2 * \mathrm{Dtoco}^{2}$
b) $\mathrm{DAP}=\mathrm{b} 0+\mathrm{b} 1 * \mathrm{Dtoco}^{2}$
d) $\mathrm{DAP}=\mathrm{b} 0+\mathrm{b} 1 *(1 / \mathrm{Dtoco})$

De posse da Análise de Variância significativa para cada um dos modelos volumétricos testados; a seleção do melhor modelo baseou-se nos seguintes critérios estatísticos: erro padrão da estimativa (Sxy) na escala da variável dependente e em percentagem (\%) e coeficiente de determinação ajustado ( $R^{2}$ aj.).

$O R^{2}$ indica o quanto da variabilidade da variável DAP $(Y)$ está associada a variável diâmetro de toco $(X)$ dentro da equação, $R^{2}=S Q R e g / S Q T o t a l$, quanto mais próximo de 1 for o valor de $R^{2}$, melhor terá sido o ajuste, mais deve-se ter muito cuidado pois quando o número de variáveis independentes varia nas equações o $R^{2}$ e tendencioso, neste caso trabalha-se com o $R^{2}$ ajustado, dado por: $1-\left\{\left(1-R^{2}\right)^{*}((n-1) /(n-p))\right\}$, o que é o caso do presente trabalho.

Tabela 1. Quadro de Análise de Variância

\begin{tabular}{ccccc}
\hline Fonte de Variação & $\begin{array}{c}\text { Grau de } \\
\text { Liberdade }\end{array}$ & Soma Quadrado & Quadrado Médio & F \\
\hline Regressão & $\mathrm{p}-1$ & SQReg. & $Q M \operatorname{Re} g=\frac{S Q \operatorname{Re} g}{G L \operatorname{Re} g}$ & $Q M \operatorname{Re} g$ \\
Resíduo & $\mathrm{n}-\mathrm{p}$ & SQRes. & $Q M \operatorname{Re} s=\frac{S Q \operatorname{Re} s}{G L \operatorname{Re} s}$ & $Q M \operatorname{Re} s$ \\
\hline Total & $\mathrm{n}-1$ & SQTotal & \\
\hline $\mathrm{p}=\mathrm{n}$ o de coeficientes do modelo testado; $\mathrm{n}=\mathrm{n}$ o de observações, árvores cubadas.
\end{tabular}


Onde:

SQTotal $=\Sigma(Y i-Y m)^{2}$ - Soma do Quadrado Total

SQReg $=\Sigma(Y e-Y m)^{2}-$ Soma do Quadrado da Regressão

SQRes $=\sum(Y i-Y e)^{2}-$ Soma do Quadrado do Resíduo

$\mathrm{Yi}=\mathrm{DAP}$ observado, real,

Ym = DAP médio observado

Ye = DAP estimado pela equação dendrometrica ajustada,

$\mathrm{n}=$ número de observações

$\mathrm{p}=$ número de coeficientes do modelo matemático ajustado.

QM Reg = Quadrado Médio da Regressão

QM Res = Quadrado Médio do Resíduo

a) Erro padrão da estimativa (Syx) na escala da variável dependente e em percentagem (Syx\%), sendo que, mais próximo de zero, mais preciso, pois o valor indica o quão próximo os valores estimados estão dos valores observados, e é obtido por meio das seguintes formulas:

$$
S y x=\sqrt{Q M \operatorname{Re} s} \quad S y x \%=\frac{S y x}{Y m} * 100
$$

Onde:

Syx $=$ Erro padrão da estimativa em termos absoluto

Syx\% = Erro padrão da estimativa em porcentagem

QM Res = Quadrado Médio do Resíduo, vide Quadro da Análise de Variância.

\section{RESULTADOS}

Na Tabela 2, observa-se as estatísticas de ajuste e precisão dos quatro modelos ajustados.

Tabela 2. Estatística de ajuste e precisão para os modelos

\begin{tabular}{|c|c|c|c|c|c|}
\hline \multirow{2}{*}{ Espaçamento } & \multirow{2}{*}{ Modelos Matemáticos } & \multicolumn{4}{|c|}{ Estatística de ajuste e precisão para modelos } \\
\hline & & $\mathrm{R}^{2}$ & $\mathrm{R}^{2}$ Ajustado & Syx & Syx\% \\
\hline \multirow{4}{*}{$3 \times 1 \mathrm{~m}$} & a) & 0,949 & 0,947 & 0,356 & 4,257 \\
\hline & b) & 0,912 & 0,910 & 0,464 & 5,553 \\
\hline & c) & 0,954 & 0,952 & 0,340 & 4,062 \\
\hline & d) & 0,903 & 0,901 & 0,488 & 5,834 \\
\hline \multirow{4}{*}{$3 \times 2 \mathrm{~m}$} & a) & 0,931 & 0,930 & 0,418 & 4,392 \\
\hline & b) & 0,887 & 0,884 & 0,537 & 5,649 \\
\hline & c) & 0,938 & 0,935 & 0,403 & 4,236 \\
\hline & d) & 0,880 & 0,877 & 0,553 & 5,819 \\
\hline \multirow{4}{*}{$3 \times 4 \mathrm{~m}$} & a) & 0,880 & 0,876 & 0,315 & 2,847 \\
\hline & b) & 0,873 & 0,870 & 0,324 & 2,924 \\
\hline & c) & 0,881 & 0,874 & 0,318 & 2,869 \\
\hline & d) & 0,876 & 0,873 & 0,320 & 2,889 \\
\hline
\end{tabular}

As estatísticas de ajuste apresentaram melhores valores para os modelos ajustados no Espaçamento $3 \times 1 \mathrm{~m}$, em média 0,930, seguido do espaçamento $3 \times 2 \mathrm{~m}$ e $3 \times 4 \mathrm{~m}, 0,909$ e 0,878, respectivamente; entretanto, todos apresentaram mais de $88 \%$; ou seja, a variável independente explica mais de $88 \%$ a variável dependente DAP. O Erro padrão da estimativa (Syx\%) indica que os valores preditos ou estimados da variável Y (DAP) estão próximos de seus respectivos valores observados ou reais; tendo sido os menores erros encontrados nos modelos ajustados para o 
espaçamento $3 \times 4 \mathrm{~m}$, em média $2,88 \%$; enquanto nos espaçamentos $3 \times 1 \mathrm{~m}$ e $3 \times 2 \mathrm{~m}$, apresentou erro médio de 4,93 e 5,02\%, respectivamente.

Os modelos "a" e "c" apresentaram nos 3 espaçamentos os menores Syx\% e os maiores $\mathrm{R}^{2}$ e $R^{2}$ ajustado, indicando uma superioridade em relação aos modelos " $b$ " e " $d$ ".

A Tabela 3 mostra os coeficientes encontrados para cada um dos modelos utilizados.

Tabela 3. Coeficientes encontrados nos ajustes

\begin{tabular}{ccccc}
\hline Espaçamento & Coeficientes a) & Coeficientes $\mathrm{b})$ & Coeficientes $\mathrm{c}$ ) & Coeficientes d) \\
\hline \multirow{3}{*}{$3 \times 1 \mathrm{~m}$} & $\mathrm{~b} 0=0,608$ & $\mathrm{~b} 0=4,480$ & $\mathrm{~b} 0=-1,598$ & $\mathrm{~b} 0=15,102$ \\
& $\mathrm{~b} 1=0,822$ & $\mathrm{~b} 1=0,042$ & $\begin{array}{c}\mathrm{b} 1=1,305 \\
\mathrm{~b} 2=-0,025\end{array}$ & $\mathrm{~b} 1=-61,028$ \\
\hline \multirow{2}{*}{$3 \times 2 \mathrm{~m}$} & $\mathrm{~b} 0=0,088$ & $\mathrm{~b} 0=4,996$ & $\mathrm{~b} 0=-2,469$ & $\mathrm{~b} 0=17,966$ \\
& $\mathrm{~b} 1=0,838$ & $\mathrm{~b} 1=0,035$ & $\mathrm{~b} 1=1,290$ & $\mathrm{~b} 1=-92,603$ \\
\hline \multirow{3}{*}{$3 \times 4 \mathrm{~m}$} & $\mathrm{~b} 0=1,707$ & $\mathrm{~b} 0=6,398$ & $\mathrm{~b} 0=-1,625$ & $\mathrm{~b} 0=20,319$ \\
& $\mathrm{~b} 1=0,713$ & $\mathrm{~b} 1=0,027$ & $\mathrm{~b} 1=1,222$ & $\mathrm{~b} 1=-120,502$ \\
\hline
\end{tabular}

A Tabela 4 mostra os resultados dos Testes de Comparação das Médias por Tukey a $5 \%$ de probabilidade, após as equações dendrometricas ajustadas terem sido aplicadas as 10 árvores não utilizadas no ajuste dos modelos e que tiveram seu DAP real mensurado em campo para validação das equações.

Tabela 4. Resultados dos Testes de Comparação de Média de Tukey a 5\% de probabilidade.

\begin{tabular}{cccc}
\hline \multirow{2}{*}{ Tratamento } & \multicolumn{3}{c}{ Espaçamento } \\
\cline { 2 - 4 } & $3 \times 1 \mathrm{~m}$ & $3 \times 2 \mathrm{~m}$ & $3 \times 4 \mathrm{~m}$ \\
\hline DAP real & $8,44000 \mathrm{a}$ & $10,18000 \mathrm{a}$ & $11,21000 \mathrm{a}$ \\
DAP est. Modelo a) & $8,51153 \mathrm{a}$ & $10,15238 \mathrm{a}$ & $11,22198 \mathrm{a}$ \\
DAP est. Modelo b) & $8,40157 \mathrm{a}$ & $10,10854 \mathrm{a}$ & $11,22107 \mathrm{a}$ \\
DAP est. Modelo c) & $8,61531 \mathrm{a}$ & $10,23852 \mathrm{a}$ & $11,28858 \mathrm{a}$ \\
DAP est. Modelo d) & $8,68517 \mathrm{a}$ & $10,16735 \mathrm{a}$ & $11,26165 \mathrm{a}$ \\
\hline DMS & 1,05238 & 1,46578 & 0,66107 \\
CV\% & 9,70 & 11,34 & 4,63 \\
\hline
\end{tabular}

Onde: DAP est. - DAP estimado com o uso da equação dendrometrica obtida com o ajuste dos modelos.

\section{DISCUSSÕES}

Com relação as estatísticas de ajuste $R^{2}$ e $R^{2}$ ajustado este trabalho encontrou valores médios superiores aos que Urban et al. (2010) encontrou para trabalho semelhante com Pinus taeda na região de Irati - PR, que foi de no máximo $88 \%$ para os mesmos modelos utilizados. Com relação as estatísticas de precisão este trabalho também encontrou valores menores que os erros encontrados por Urban et al. (2010) que foi em média de $6,89 \%$, enquanto este trabalho encontrou $2,88 \%, 4,93 \%$ e 5,02\%, para os respectivos espaçamentos analisados, $3 \times 4 \mathrm{~m}$, 3x1m e $3 \times 2 m$.

\section{CONCLUSÕES}

Neste estudo pode-se concluir que todos os modelos utilizados conseguem explicar a variável independente DAP em função do diâmetro dos tocos; e os DAP estimados com o uso das 
equações dendrometricas ajustadas, diferentes modelos, não diferiram estatisticamente entre si; ou seja, qualquer modelo pode ser utilizado nos diferentes espaçamentos para estimar o DAP em função do diâmetro do toco, mesmo que os modelos " $a$ " $e$ " $c$ " tenham apresentado melhores estatísticas de ajuste e precisão quando comparado entre si.

\section{REFERÊNCIAS}

LEITE, F. S. Estimativa do volume de madeira a partir do diâmetro da cepa em uma área explorada de floresta amazônica de terra firme. 2008. 74 f. Dissertação (Mestrado em Ciências Florestais) Universidade de Brasília, Brasília, 2008.

MACHADO, S. A.; FIGUEIREDO FILHO, A. Dendrometria. Guarapuava: UNICENTRO. 2 Ed., 2006. $316 p$.

NASCIMENTO, R. G. M.; MACHADO, S. do A.; FIGUEIREDO, D. J. de; AUGUSTYNCZIK, A. L. D.; CAVALHEIRO, R. Relações dendrométricas de Araucaria angustifólia. Pesquisa Florestal Brasileira, Colombo, v. 30, n.64, p. 369-374, nov./dez. 2010.

SOARES, C. P. B., NETO, FRANCISCO de P. e SOUZA, A. L. de. Dendrometria e Inventário Florestal. Viçosa: Ed. UFV, 2006. 276p.:il.

URBAN, C. W.; LISBOA, G. dos S.; STEPKA, T. F.; KURCHAIDT, S. M. Estimativa do DAP em função do diâmetro do toco para plantios de Pinus taeda na região de Irati - PR. Anais do XIX EAIC. Guarapuava: UNICENTRO. 2010. 